therapy group were found to be significantly lower $(-0.41 \pm 0.29 \mathrm{mg} / \mathrm{dL}, \mathrm{P}<0.001)$. However, the serum uric acid levels in the estrogen monotherapy and tibolone groups did not differ significantly from the control group level.

Conclusions: Serum uric acid levels decreased in response to estrogenprogestogen combination therapy in postmenopausal women. We attribute our findings to the effects of progesterone, rather than estrogen.

References:

[1] Roddy E, Doherty M. Epidemiology of gout. Arthritis Res Ther. 2010;12:223.

[2] Hak AE, Curhan GC, Grodstein F, Choi HK. Menopause, postmenopausal hormone use and risk of incident gout. Ann Rheum Dis. 2010;69:1305-9.

[3] Hak AE, Choi HK. Menopause, postmenopausal hormone use and serum uric acid levels in US women-the Third National Health and Nutrition Examination Survey. Arthritis Res Ther. 2008;10:R116.

[4] Bruderer SG, Bodmer M, Jick SS, Meier CR. Association of hormone therapy and incident gout: population-based case-control study. Menopause. 2015;22:1335-42.

Acknowledgements: No grants or other support were received for this study.

Disclosure of Interest: None declared

DOI: 10.1136/annrheumdis-2017-eular.1950

\section{SAT0694 SUBGROUPING OF EARLY RA-PATIENTS IDENTIFIED CLUSTER OF PATIENTS WITH HIGH LEVELS OF PAIN, FATIGUE AND PSYCHOSOCIAL DISTRESS 3 YEARS AFTER DIAGNOSIS}

J. Lindqvist ${ }^{1}$, H. Westerlind ${ }^{2}$, L. Klareskog ${ }^{1}$, L. Alfredsson ${ }^{2}$, J. Lampa ${ }^{1} .{ }^{1}$ Unit of Rheumatology, Department of Medicine Solna; ${ }^{2}$ Institute of Environmental Medicine, Karolinska Institute, Stockholm, Sweden

Background: The wide range of effective treatment alternatives for rheumatoid arthritis (RA) makes treating the disease to inflammatory remission a feasible goal for a majority of patients. However, earlier studies have reported that symptoms other than inflammatory disease activity causes a substantial burden of illness for RA-patients. These unmet needs include persistent pain, fatigue, impaired physical function and mental health status (1).

Objectives: To identify clusters of early RA-patients based on pain, fatigue, sleep, physical function, mental health status and perceptions of quality of life, 3 years after diagnosis. Withal investigate associations between clusters and clinical parameters at the time of diagnosis.

Methods: Data was compiled from the Swedish case-control cohort Epidemiological Investigation of Rheumatoid Arthritis (EIRA) and linked to the Swedish Rheumatology Quality Register (SRQ). All patients were diagnosed with RA according to the 1987 ACR criteria. Early RA-patients with clinical data from diagnosis and 3 year follow-up questionnaire data were included (N=618; 74\% women, median age at diagnosis 58 years). Measurements of pain, fatigue, sleep problems, physical and mental functioning and quality of life was entered into a hierarchical agglomerative clustering procedure using Ward's method of squared Euclidian distances. Number of clusters was determined by largest changes in distances at which clusters were formed. Associations between clusters and clinical variables at diagnosis were calculated using contingency analysis. All statistical analysis was performed using jmp statistical software (SAS, US). $\mathrm{p}<0.05$ was considered significant.

Results: The cluster analysis identified three distinct clusters. Cluster 1 consisted of 178 patients $(29 \%)$ doing significantly worse for all included variables. Cluster 3 consisted of 209 patients (34\%) doing very well and cluster 2, consisting of 231 patients $(37 \%)$, constituted an intermediate group doing fairly well. Cluster 1 was associated with female sex $(p=0.0007)$ and lower education level $(p=0.0003)$ compared to cluster 3 . Cluster 1 was also associated to higher HAQ $(p<0,0001)$, higher patient global assessment of health $(p<0.0001)$, higher pain ratings $(p<0.0001)$ and lower swollen/tender joint count ratio (STR) $(p=0.0065)$ at the time of diagnosis compared to cluster 3 .

Conclusions: Through cluster analysis, we could identify a subgroup of almost a third of the RA-patients with high levels of pain, fatigue, sleep problems and poor physical and mental health related quality of life 3 years after RA-diagnosis. These symptoms are indicative of a central sensitization syndrome and these findings indicate that other factors than inflammatory disease activity causes a significant burden of illness also at an early stage of RA and that there is a need of additional intervention strategies for these patients.

References:

[1] Taylor PC, Moore A, Vasilescu R, Alvir J, Tarallo M. A structured literature review of the burden of illness and unmet needs in patients with rheumatoid arthritis: a current perspective. Rheumatol Int. 2016;36(5):685-95.

Acknowledgements: All patients and staff involved in EIRA ans SRQ.

Disclosure of Interest: None declared

DOI: 10.1136/annrheumdis-2017-eular.3740

\section{SAT0695 NO ASSOCIATION BETWEEN VITAMIN D LEVELS AND CARDIOVASCULAR DISEASES IN INFLAMMATORY JOINT DISEASES AND SYSTEMIC AUTOIMMUNE DISEASES - A SYSTEMATIC REVIEW}

J.E.S. Ling ${ }^{1}$, M. Ter Wee ${ }^{2}$, W. Lems ${ }^{3}$, M. Nurmohamed ${ }^{3}$, H. Raterman ${ }^{3}$. ${ }^{1}$ Newcastle University Medicine Malaysia, Johor Bahru, Malaysia; ${ }^{2}$ Epidemiology and Statistics; ${ }^{3}$ Amsterdam Rheumatology and Immunology Centre, VU University Medical Centre, Amsterdam, Netherlands

Background: In recent years, vitamin $D$ deficiency has been linked to disease activity and pathogenesis of systemic autoimmune diseases (SAD) like systemic lupus erythematosus (SLE) and inflammatory joint diseases (IJD) such as rheumatoid arthritis (RA). In the general population, the association between vitamin D with risk for cardiovascular diseases (CVD) is still debatable. While people with IJD and SAD tend to be vitamin D deficient and suffer from an elevated CVD burden, the effect of vitamin $D$ on their cardiometabolic risk factors is of much interest.

Objectives: To provide an overall conclusion on whether vitamin $D$ deficiency contributes to an increased cardiovascular morbidity in these patients, a systematic literature review was done.

Methods: A systematic literature search was done in PubMed/MEDLINE and EMBASE to identify all articles that assessed the association of vitamin D with cardiovascular disease and its risk factors in patients with IJD (rheumatoid arthritis (RA), psoriatic arthritis (PsA), ankylosing spondylitis) and SAD (systemic lupus erythematosus (SLE), Behcet's disease, vasculitis, Sjogren syndromes, systemic sclerosis). Eligible studies were assessed for quality and risk of bias according to the Cochrane Handbook Chapter 13.5.2.1. (Higgins JPT. GS. Cochrane Handbook for Systematic Review of Interventions The Cochrane Collaboration 2011.)

Results: In total 3273 abstracts were identified. After screening, selection and quality assessment, 16 studies were included (6 case-control and 10 cohort studies), which described only RA and SLE except for one study which focused on PsA. Therefore, this study focused on RA and SLE because they are the most frequent IJD and with highest CVD risk respectively. In RA patients $(n=812)$ vitamin $\mathrm{D}$ deficiency was associated with presence of (components) of metabolic syndrome $(\mathrm{OR}=1.8(95 \% \mathrm{Cl}: 1.3 ; 2.5), \mathrm{P}=0.001)$ in $\mathrm{RA}$, especially dyslipidemia (OR 1.7; 95\% Cl:1.1-2.5; $\mathrm{P}=0.013$ ) and obesity. No studies with prospective design in RA have assessed CVD risk in relation to vitamin D. In SLE patients $(n=1850)$ the only prospective study observed no association between vitamin D deficiency and CVD, although weak associations with dyslipidemia and obesity were observed in some studies.

Conclusions: No clear association between vitamin D deficiency and CVD was found in patients with RA and SLE, probably due to large heterogeneity in terms of sample sizes, designs, analyses and outcome measures. As conclusions were mainly drawn on cross-sectional data, there is an urgent need for adequate prospective studies to assess if vitamin D levels are associated with cardiovascular outcomes.

\section{References:}

[1] Higgins JPT, Green S (editors). Cochrane Handbook for Systematic Reviews of Interventions Version 5.1.0 [updated March 2011]. The Cochrane Collaboration, 2011. Available from www.handbook.cochrane.org.

Disclosure of Interest: None declared

DOI: 10.1136/annrheumdis-2017-eular.4302

\section{SAT0696 ENDOTHELIAL NITRIC OXIDE SYNTHASE T-786C GENE PROMOTER POLYMORPHISM IS A POTENTIAL PREDICTOR OF LOW RESPONSE TO THE THERAPY IN PATIENTS WITH RHEUMATOID ARTHRITIS, UKRAINE POPULATION}

K. Zaichko, M. Stanislavhuk, I. Shapoval. The Internal Medicine, National Pirogov Memorial Medical University, Vinnytsya, Ukraine

Background: Recently endothelial nitric oxide synthase (eNOS) T-786C gene promoter polymorphism was considered to be a factor of a high severity of RA $[1,2]$. It is possible that the eNOS T-786C gene promoter polymorphism can modify the efficacy and safety of treatment in patients with RA, but there is no information on the subject.

Objectives: To evaluate efficacy of treatment of patients with RA in accordance to the eNOS T-786C gene promoter polymorphism.

Methods: Patients who were enrolled in the study satisfied follows criteria: MTX 10-15 mg/week during the past 12 weeks, stable oral NSAIDs and corticosteroids (CCS), $\leq 10 \mathrm{mg} /$ day prednisone or equivalent during the past 2 weeks, DAS28 $\geq 3.2,>6$ tender and swollen joints. We excluded patients with previous biologic agent treatment history. All patients received treatment that included MTX, folic acid, CCS, NSAIDs. We evaluated RA activity (DAS28), number of swollen and tender joints, ESR, CRP, and HAQ before enrollment in the study and at the 12 week. Efficacy of the treatment was assessed by the ACR 20;50; 70 at the end of the study.

The 12-week study completed 148 patients with RA, $100 \%$ female, aged $45.7 \pm 8.54$ years (mean \pm SD), with moderate (DAS28 $3.2-5.1 ; 34.5 \%$ ) and high disease activity (DAS28 $>5.1 ; 65.5 \%$ ). Among the patients with RA, seropositive were $81.8 \%$ and $84.5 \%$ by the RF and the anti-CCP, respectively. Polymorphism of NOS3 T786C gene (rs2070744) was performed by Real-Time PCR. All studied polymorphism satisfied Hardy-Weinberg equilibrium. The study was conducted in accordance with the Declaration of Helsinki of the World Medical Association 
"Ethical principles of medical research involving human subjects" (2000), the requirements of GCP, the applicable national legislation.

Results: Among patients with RA frequency of the genotypes was as follows: TT $-37.2 \%$, TC $-42.6 \%$, CC $-20.3 \%$. Age, seropositivity and the duration of the disease were not differing in patients with RA, carriers of the different genotypes of eNOS. Though, CC genotype was associated with high disease activity according to DAS28-ESR $>5.2$ (OR=9.60; 95\% Cl 2.18-42.2), $\mathrm{HAQ}>2$ $(\mathrm{OR}=2.38 ; 95 \% \mathrm{Cl}: 0.94-6.02)$ and extraarticular manifestations (OR $=3.26 ; 95 \%$ Cl 1.40-7.56).

After 12 weeks treatment we estimated, that among patients with TT genotype there were 58.3; 16.7; and 8.3\% responders ACR20; ACR50; ACR70, and among patients with $C C$ genotype $-20.0 ; 10.0 ; 0.0 \%(p<0.05)$. TC heterozygotes patients had lower (by $22-25 \%, \mathrm{p}<0.05$ ) clinical response to the treatment by DAS28 and $\mathrm{HAQ}$ than the homozygotes TT.

Conclusions: eNOS T-786C gene promoter polymorphism influence on the efficacy of the treatment, and CC genotype can be considered as a possible predictor of a low response to the treatment in patients with rheumatoid arthritis. References:

[1] Breedveld F.C., Weisman M.H., Kavanaugh A.F. et al. (2006) The PREMIER study. Arthr. Reum., 54: 26-37.

[2] Gonzalez-Gay M.A., Llorca J., Palomino-Morales R. et al. (2009) Influence of nitric oxide synthase gene polymorphisms on the risk of cardiovascular events in rheumatoid arthritis. Clin. Exp. Rheumatol., 27 (1): 116-119.

Disclosure of Interest: None declared

DOI: 10.1136/annrheumdis-2017-eular.3962

\section{SAT0697 PREVALENCE OF HEPATITIS B AND C INFECTION AND REACTIVATION IN PATIENTS RECEIVING BIOLOGIC IV THERAPIES}

K. Arévalo Ruales ${ }^{1}$, B. Soler Bas ${ }^{2}$, J. Ivorra Cortes ${ }^{1}$, E. Grau Garcia ${ }^{1}$, E. Vicens Bernabeu $^{1}$, E. Labrador Sánchez ${ }^{1}$, J.J. Fragio Gil ${ }^{1}$, R. González Mazarío ${ }^{1}$, C. Alcañiz Escandell ${ }^{1}$, I. Cánovas Olmos ${ }^{1}$, I. Chalmeta Verdejo ${ }^{1}$, C.M. Feced Olmos $^{1}$, L. Gonzalez Puig ${ }^{1}$, I. Martínez Cordellat ${ }^{1}$, C. Nájera Herranz ${ }^{1}$, R. Negueroles Albuixech ${ }^{1}$, J.E. Oller Rodriguez ${ }^{1}$, F.M. Ortiz-Sanjuan ${ }^{1}$, J.A. Román Ivorra ${ }^{1,2} .{ }^{1}$ Rheumatology Department, HUP la Fe; ${ }^{2}$ Medical School, UCV, Valencia, Spain

Background: With the use of biological therapy, cases of reactivation of hepatitis $B$ (HBV) and C (HCV) have been described, consequently its use has to be carefully evaluated. In some cases these therapies have to be administered together with antiviral treatment. Despite the fact that before the beginning of the biological therapy the screening of HBV and HCV virus is done, during the course of treatment serologies are no longer used to detect serovonversions.

Objectives: To analyze the prevalence of HBV and HCV in patients with rheumatic disease at the beginning of biological therapies. To identify the cases of hepatitis reactivation.

Methods: Retrospective observational analysis of the biological treatments administered to patients with rheumatic Disease of the rheumatology department of La Fe hospital, during the period 2000-2015 who had HBV and/or HCV serology at the beginning of treatment. Demographic data and diagnosis of the patient, months of treatment and the results of the serologies performed at the beginning and in the follow-up of the treatment, and the cases of reactivation of hepatitis have been collected.

Results: A total of 388 patients were selected of which $62.4 \%$ were female; the mean age at diagnosis of was $38.96 \pm 14.36$ years. $49.5 \%$ of the patients were diagnosed with rheumatoid arthritis (RA), $25.3 \%$ of ankylosing spondylitis (AE), $18.6 \%$ of psoriatic arthritis (APSO) and $6.7 \%$ with other diagnoses. The mean treatment time with patients' biological therapy is $200 \pm 118$ months (3-464 months), and the mean time between the two serologies is $172 \pm 102$ months (18-460 months). An incidence of $10 \%$ of cases with HBV infection (39 patients) is observed. The serological pattern was 8 cases with Ac-core (+) HBV/Ac-surface $(-) /$ Ag-surface (-) HBV and 31 HBV/Ac- surface (-). The incidence of HCV at the start of treatment was $1.1 \%$ (4 patients). A case of reactivation $(12.5 \%$ ) was detected among the 8 patients with Ac-core (+)/Ac-surface (-)/Ag-surface (-) HBV. The patient had infliximab and methotrexate, and reactivation was observed after 12 years of treatment. No reactivation of HCV has been detected.

Conclusions: In our series of patients the incidence of HBV and HCV has been $10 \%$ and $1.1 \%$, respectively. One of the 8 patients with Ac-core (+)/Ac-surface $(-) /$ Ag-surface (-) HBV, had reactivation of the virus.

Disclosure of Interest: None declared

DOI: 10.1136/annrheumdis-2017-eular.4827

\section{SAT0698 DISEASE ACTIVITY DURING AND AFTER PREGNANCY IN WOMEN WITH AXIAL SPONDYLOARTHRITIS}

K. Ursin ${ }^{1,2}$, S. Lydersen ${ }^{3}$, J. Skomsvoll ${ }^{1}$, M. Wallenius ${ }^{1,2}{ }^{1}$ National Advisory Unit on Pregnancy and Rheumatic Diseases, St. Olavs Hospital - Trondheim University Hospital; ${ }^{2}$ Department of Neuromedicine and Movement Science; ${ }^{3}$ Regional Centre for Child and Youth Mental Health, NTNU, Norwegian University of Science and Technology, Trondheim, Norway

Background: Studies on disease activity of ankylosing spondylitis in pregnancy have shown diverging results. A large retrospective study from 1998 without validated disease activity scores found no particular pattern of disease course during pregnancy (1). Later two small studies demonstrated a tendency towards lower disease activity in the beginning of pregnancy and deterioration in late pregnancy $(2,3)$. None of these studies included women with non-radiographic axial spondyloarthritis. The only large study was conducted before the widespread use of biological DMARDs.

Objectives: The aim of this project was to prospectively study disease activity in women with axial spondyloarthritis before, during and after pregnancy with BASDAI as disease activity measure.

Methods: RevNatus is a Norwegian nationwide register designed for the followup of pregnant women with rheumatic diseases. RevNatus included 181 full term pregnancies in 168 women with axial spondyloarthritis between 2006 and 2016. The women had seven visits at a rheumatology unit; before pregnancy, in each trimester, and six weeks, six months and twelve months postpartum. BASDAl-values from each visit were analyzed in a linear mixed model.

Results: Even though we found a statistically significant relationship between disease activity and time point in the follow-up period, our study demonstrated that women with axial spondyloarthritis had stable, low disease activity during and after pregnancy. Disease activity in second trimester was significantly higher than six weeks after delivery, but the change in estimated mean BASDAI was small (BASDAI 3.97 vs. BASDAI $3.46, p=0.005$ ). The figure below shows changes in disease activity throughout the study period.

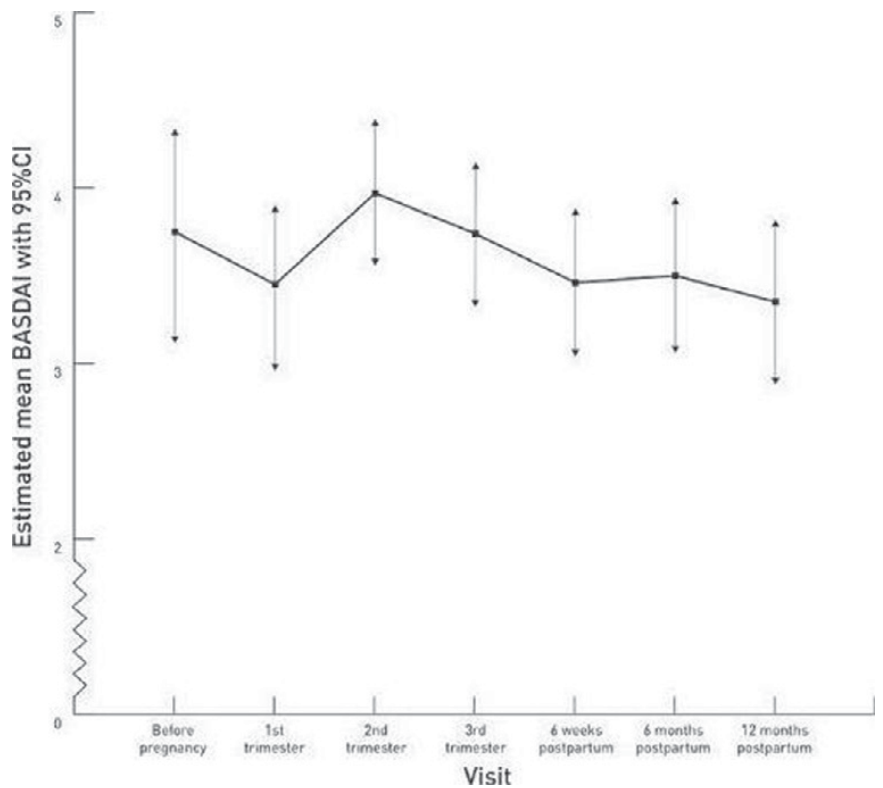

Conclusions: Studying women with axial spondyloarthritis, we found that disease activity was highest in second trimester, but altogether stable in the period from planning pregnancy to one year after delivery.

References:

[1] Ostensen M, Ostensen H. Ankylosing spondylitis - The female aspect. J Rheumatol. 1998;25(1):120-4.

[2] Ostensen M, Fuhrer L, Mathieu R, Seitz M, Villiger PM. A prospective study of pregnant patients with rheumatoid arthritis and ankylosing spondylitis using validated clinical instruments. Ann Rheum Dis. 2004;63(10):1212-7.

[3] Lui NL, Haroon N, Carty A, Shen H, Cook RJ, Shanmugarajah S, et al. Effect of pregnancy on ankylosing spondylitis: A case-control study. J Rheumatol. 2011;38(11):2442-4.

Disclosure of Interest: None declared

DOI: 10.1136/annrheumdis-2017-eular.1604

\section{SAT0699 RAPID ASSESSMENT PREDICTS DISEASE ACTIVITY IMPROVEMENT IN NEWLY DIAGNOSED RHEUMATOID ARTHRITIS (RA)}

M. Yates ${ }^{1}$, J. Galloway ${ }^{1}$, N. Snowden ${ }^{2}$, S. Norton ${ }^{1}$, J.M. Ledingham ${ }^{3}$ E.M. Dennison ${ }^{4}$, A. Rivett ${ }^{5}$, A.J. Macgregor ${ }^{6} .{ }^{1}$ Academic Rheumatology, King's College London, London; ${ }^{2}$ Rheumatology Dept, Pennine MSK Partnership, Oldham; ${ }^{3}$ Rheumatology Dept, Portsmouth Hospitals NHS Trust, Portsmouth; ${ }^{4} M R C$, Southampton University, Southampton; ${ }^{5} B S R$, The British Society for Rheumatology, London; ${ }^{6}$ Rheumatology Dept, Norfolk and Norwich University Hospital, Norwich, United Kingdom

Background: Early intervention in RA is associated with improved outcomes in randomised trials. UK guidelines stipulate that those with suspected RA are assessed by a rheumatologist within 3 weeks of referral. However, there are limited real world data confirming the value of early assessment. Previous work suggests social deprivation predicts severe disease at presentation and a worse 